\title{
Endoscopic Findings of Enteropathy-Associated T-Cell Lymphoma Type II: A Case Series
}

Yun Soo Hong*, Young Sik Woo ${ }^{\dagger}$, Gaeun Park*, Kyungho Lee*, Soo Hoon Kang*, Hyun Woo Lee*, Eun Ran Kim ${ }^{\dagger}$, Sung Noh Hong ${ }^{\dagger}$, Dong Kyung Chang ${ }^{\dagger}$, Young Ho Kim ${ }^{\dagger}$, Poong-Lyul Rhee ${ }^{\dagger}$, and Jae J. Kim ${ }^{\dagger}$

${ }^{*}$ Department of Medicine and ${ }^{\dagger}$ Division of Gastroenterology, Department of Medicine, Samsung Medical Center, Sungkyunkwan University School of Medicine, Seoul, Korea

Enteropathy-associated T-cell lymphoma (EATL) is a rare extranodal T-cell lymphoma arising from the intestine. Two types of EATL have been reported. In contrast to the classic EATL type I, EATL type II occurs sporadically, is unrelated to celiac disease, and comprises $10 \%$ to $20 \%$ of all EATL cases. A total of five cases of EATL type II were diagnosed at our clinic from January 2009 to September 2012. Four of the five patients were diagnosed with the help of endoscopy. Among the four patients, two of the cases involved both the small and large intestines, whereas in the other two patients, EATL was limited to the small intestine. Common endoscopic findings included innumerable fine granularities (also called mosaic mucosal patterns) and diffuse thickening of the mucosa with a semicircular shallow ulceration in the lesions of the small bowel. In contrast, the endoscopic findings of the colon were nonspecific and could not distinguish EATL type II from other diseases. There are only few published reports regarding the representative endoscopic findings of EATL. Here, we present the clinical and endoscopic findings of four cases of EATL type II diagnosed by endoscopy. (Gut Liver 2016;10:147-151)

Key Words: Enteropathy-associated T-cell lymphoma type II; Gastrointestinal lymphoma; Endoscopy; Double-balloon enteroscopy

\section{INTRODUCTION}

Enteropathy-associated T-cell lymphoma (EATL) is a rare intestinal tumor, accounting for less than 5\% of gastrointestinal lymphoma. It is known to arise from $\mathrm{T}$ lymphocytes that reside in the intraepithelial space of the intestines and is classified into two types. ${ }^{1}$ The classic EATL, also known as EATL type I, is predominantly found in Western countries and is strongly associated with refractory celiac disease. In this type, tumor cells are large and pleomorphic and positive for CD3 and CD7 in immunohistochemistry staining, but negative for CD4, CD8, or CD56. The other type, namely EATL type II, comprises 10\% to 20\% of all EATL cases. EATL type II is mainly composed of small to medium-sized cells, which show positivity for CD3, CD7, CD8, and CD56. It develops sporadically and is independent of celiac disease. ${ }^{2}$ EATL is most frequently found in the small intestine, especially the proximal jejunum, followed by stomach, colon, and rectum. It is clinically associated with symptoms of malabsorption and is typically diagnosed upon laparotomy, as the first presenting signs are usually spontaneous intestinal perforation and/or obstruction. ${ }^{3}$ Currently, only few published reports are available regarding the endoscopic findings of EATL type II distinct from classic EATL. Here, we present four cases of EATL type II diagnosed preoperatively with the endoscopy.

\section{CASE REPORTS}

Five cases of EATL type II were diagnosed from January 2009 to September 2012 at Samsung Medical Center. Of the five cases, four were diagnosed endoscopically and the other one surgically. None of the patients had a history of celiac disease or malabsorption. The patients (three men and one woman) ranged in age from 48 to 67 years (median, 52.5 years). Two patients presented with chronic diarrhea and the other two with abdominal pain. Both the small and large intestines were involved in two patients while the disease was limited to the small intestine

Correspondence to: Eun Ran Kim

Division of Gastroenterology, Department of Medicine, Samsung Medical Center, Sungkyunkwan University School of Medicine, 81 Irwon-ro, Gangnam-gu, Seoul 06351, Korea

Tel: +82-2-3410-3407, Fax: +82-2-3410-6983, E-mail: er.kim@samsung.com

Received on November 21, 2014. Revised on January 9, 2015. Accepted on February 24, 2015. Published online August 11 , 2015

pISSN 1976-2283 eISSN 2005-1212 http://dx.doi.org/10.5009/gnl14457

Yun Soo Hong and Young Sik Woo contributed equally to this work as first authors.

@ This is an Open Access article distributed under the terms of the Creative Commons Attribution Non-Commercial License (http://creativecommons.org/licenses/by-nc/4.0) which permits unrestricted non-commercial use, distribution, and reproduction in any medium, provided the original work is properly cited. 


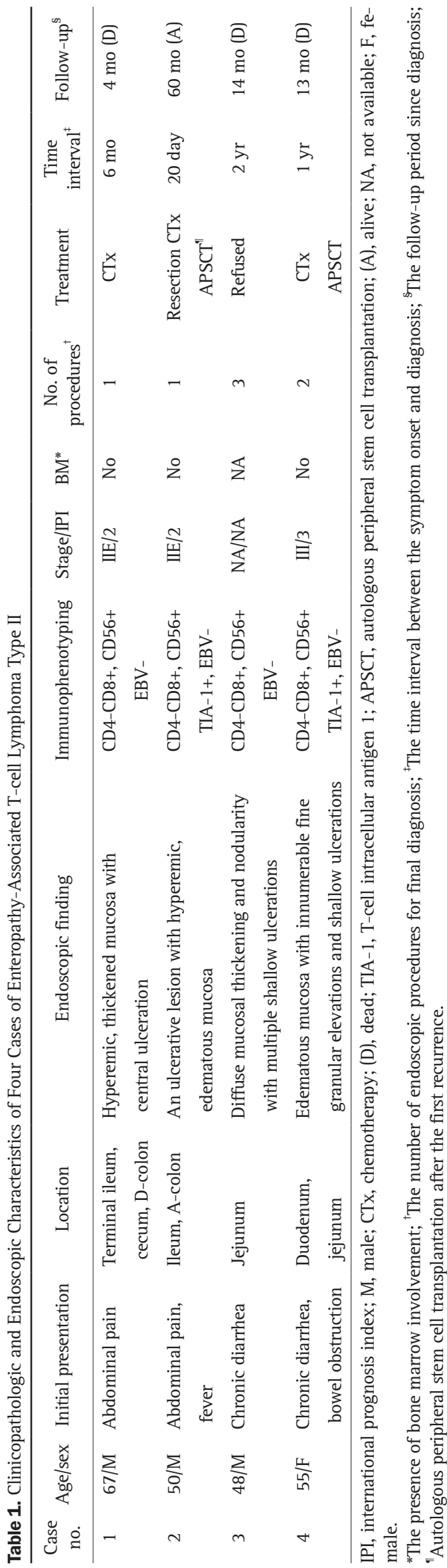

in the other two patients (Table 1).

\section{Case 1}

A 67-year-old man was admitted for evaluation of abdominal mass, night sweating and weight loss over the period of 6 months. computed tomography (CT) scanning and positron emission tomography (PET) showed a 6-cm-sized homogeneous mass on the terminal ileum and ascending colon. Colonoscopy revealed hyperemic, thickened mucosa with central ulceration on the cecum (Fig. 1A) and a 1.5-cm-sized pinkish flesh-like, flat thickened lesion on the proximal descending colon (Fig. 1B). Biopsy specimens from each lesion showed diffuse infiltration of small- to medium-sized atypical lymphoid cells with CD4-, CD8+ and CD56+ immunophenotypes. Despite conventional chemotherapy, leptomeningeal seeding was confirmed after the fourth cycle of cyclophosphamide, doxorubicin, vincristine, and prednisone (CHOP). He died of disease progression and septic shock due to pneumonia.

\section{Case 2}

A 50-year-old man was admitted with complaints of abdominal pain and low grade fever for 20 days. Colonoscopy revealed about 3-cm-sized discrete ulcerative lesion with hyperemic edematous mucosa on the proximal ascending colon (Fig. 1C). Biopsy specimens from the lesion showed diffuse infiltration of small- to medium-sized atypical lymphoid cells with CD4-, CD8+, CD56+, and T-cell intracellular antigen 1 (TIA-1)+ immunophenotypes (Fig. 2). As abdominal pain progressively worsened, he underwent urgent exploratory laparotomy. During laparotomy, bowel wall thickening on the distal ileum extended $15 \mathrm{~cm}$ in length, its severe adhesion to surrounding structures forming a mass. The involved segment including the mass was resected. The surgical specimen also was pathologically consistent with EATL type II. Successful complete remission was achieved after six cycles of rituximab, cyclophosphamide, doxorubicin, vincristine, and prednisone (R-CHOP). After 3 years, abdominal pain developed. Double-balloon-enteroscopy (DBE) revealed diffuse fine granularity with circumferential shallow ulceration in the mid-ileum (Fig. 3A) and pathological examination confirmed the recurrence of EATL. He underwent autologous peripheral blood stem cell transplantation (APBSCT) and has been free of disease for 11 months.

\section{Case 3}

A 48-year-old male was admitted with complaints of chronic diarrhea and weight loss of $14 \mathrm{~kg}$ over the course of 1 year. Although the initial esophagogastroduodenoscopy (EGD) and colonoscopy were unremarkable, CT scan revealed the focal enhancing lesion in small bowel. DBE via antegrade approaches revealed diffuse mucosal thickening and nodularity with multiple shallow semicircular ulcerations from mid to distal jejunum (Fig. 3B). Pathologic specimens from the lesions demonstrated 

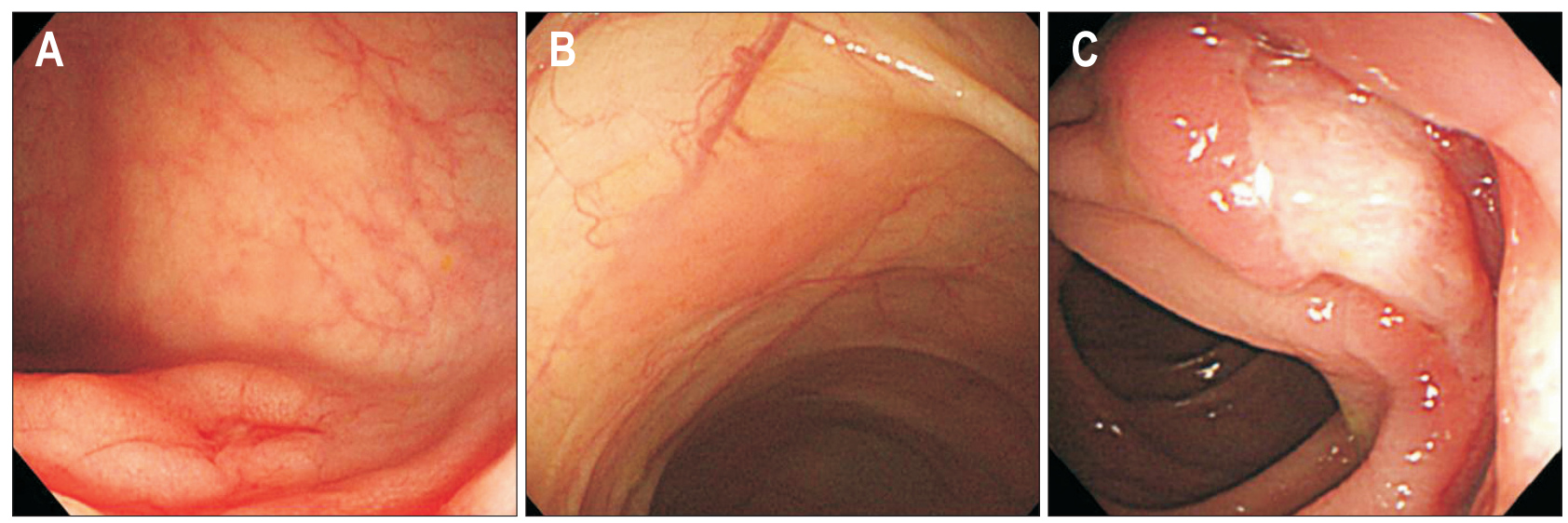

Fig. 1. Representative features of endoscopic findings of colonic enteropathy-associated T-cell lymphoma. (A) Colonoscopy revealed hyperemic, thickened mucosa with central ulceration on the cecum and (B) flesh-like flat thickened lesions on the proximal descending colon. (C) There was a discrete ulcerative lesion with diffuse mucosal thickening and edematous mucosa on the ascending colon.
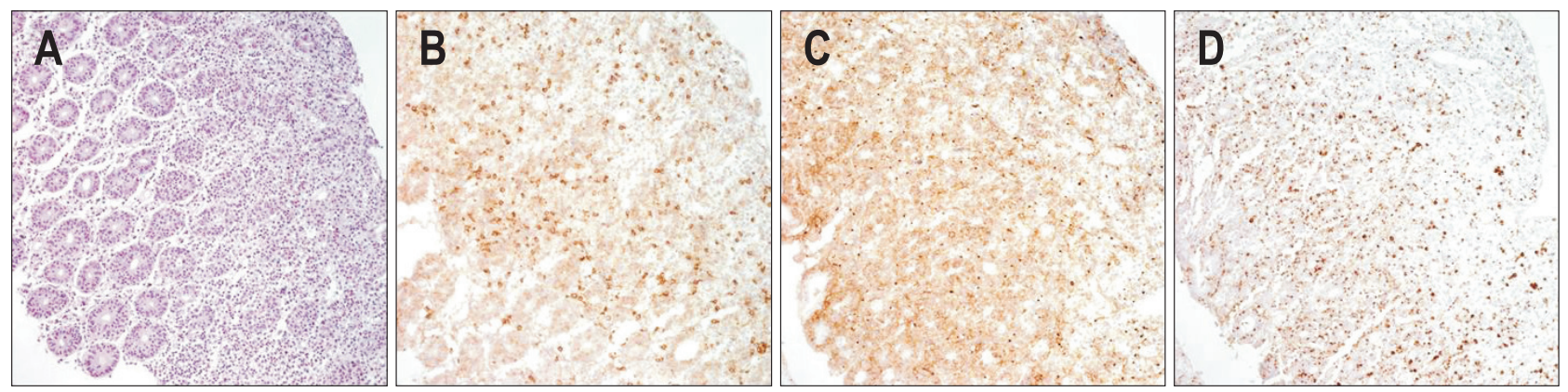

Fig. 2. Representative features of typical pathologic findings of colonic enteropathy-associated T-cell lymphoma. (A) The colon biopsy showed heavy intraepithelial lymphocytosis involving the surface epithelium (H\&E stain, ×200). Many CD8-positive (B), CD56-positive (C), and T-cell intracellular antigen 1-positive (D) intraepithelial lymphocytes were scattered $(\times 200)$.

diffuse infiltration of small- to medium-sized atypical lymphoid cells. Immunohistochemistry was negative for CD4 but positive for CD8 and CD56. After the diagnosis, the patient refused further treatment and died at 14 months after diagnosis.

\section{Case 4}

A 55-year-old woman was referred to Gastroenterology Department because of chronic diarrhea, abdominal pain, and weight loss, which persisted for 1 year. Although the initial EGD was unremarkable, abdomen and pelvic CT revealed luminal narrowing in the 3rd portion of duodenum. She was admitted for evaluation and during admission, obstructive symptoms, such as vomiting, developed. Re-evaluation with EGD showed luminal obstruction due to an encircling mass at the proximal jejunum. However, only necrotic debris was found on the pathology. Moreover, without definite 2-deoxy-2-[fluorine-18]fluoroD-glucose uptake, PET suggested inflammatory condition of the lesion rather than malignancy. During the surgery, there was diffuse swelling and luminal narrowing from the ligament of Treitz to proximal jejunem and the involved segment was $15 \mathrm{~cm}$ in length. Because surgeon judged to have no resectability, gas- trojejunostomy without duodenectomy was performed to relieve obstructive symptoms after the biopsy of enlarged lymph node. Biopsy specimens from enlarged lymph node revealed only reactive change with no evidence of malignancy. Only 2 weeks after discharge, she was re-admitted for abdominal pain, nausea, and poor oral intake. A subsequent EGD through the gastrojejunostomy site revealed shallow ulcerations which encircled the diffusely swollen lumen of the afferent loop of the jejunum (Fig. 3C). Edematous mucosa with innumerable fine granular elevations, described as velvety or sand-like mucosa, caused obstruction of the, mid to distal jejunum (Fig. 3D). Biopsy specimens from these lesions revealed diffuse infiltration of small- to medium-sized atypical lymphoid cells with CD4-, CD8+, CD56+, and TIA-1 positivity (Fig. 4). Tissue DNA analysis demonstrated monoclonal T-cell receptor $\gamma$ rearrangement. Based on these findings, the diagnosis of EATL type II was made. She was unresponsive to $\mathrm{CHOP}$ and chemotherapy regimen was changed. After ten months of therapy, she underwent APBSCT because of disease progression but during the course, she died of sepsis. 

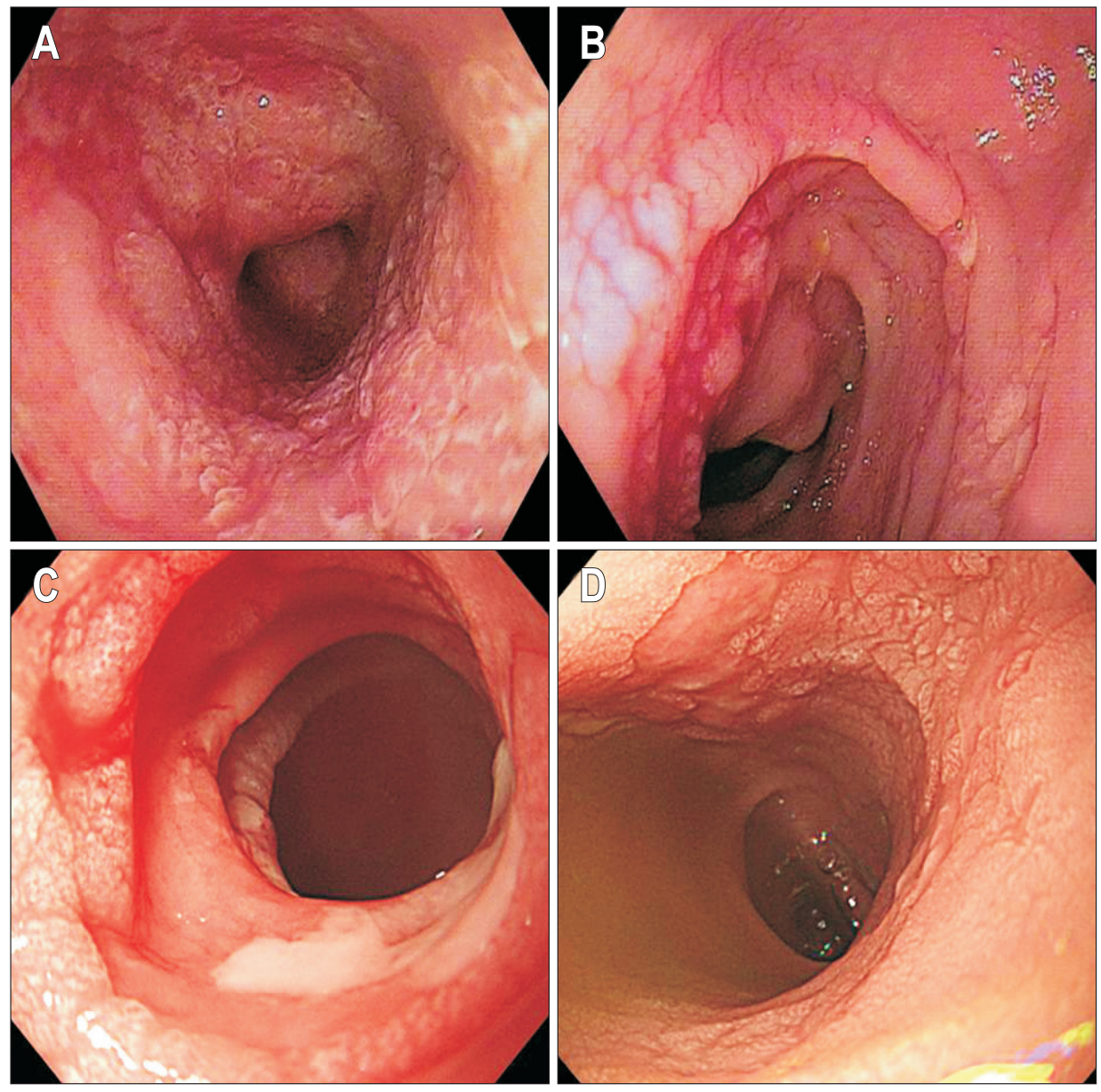

Fig. 3. Representative features of endoscopic findings of the smallbowel enteropathy-associated Tcell lymphoma. (A) Double-balloonenteroscopy (DBE) showed diffuse fine granularity and edematous mucosa with circumferential shallow ulcerations on the ileum. (B) DBE revealed diffuse mucosal thickening and nodularity with multiple shallow semicircular ulcerations from the mid to distal jejunum. In another cases, shallow ulcerations encircled the diffusely swollen mucosa of the jejunum (C), and innumerable fine granular elevations (velvety or sand-like mucosa) were observed on the mid to distal jejunum (D).
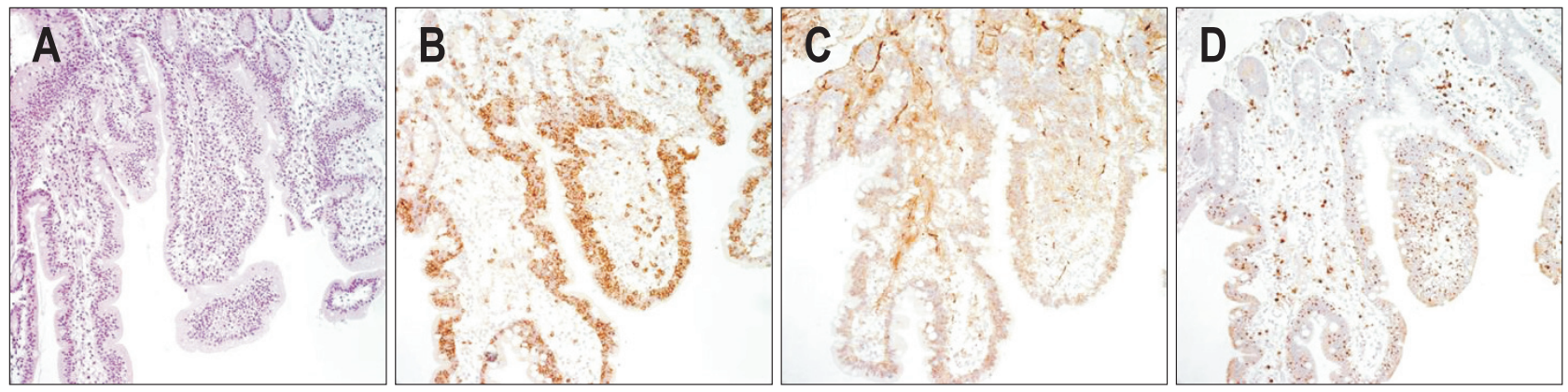

Fig. 4. Representative features of typical pathologic findings of small-bowel enteropathy-associated T-cell lymphoma. (A) The small intestine biopsy showed heavy intraepithelial lymphocytosis involving the surface epithelium (H\&E stain, ×200). Many CD8-positive (B), CD56-positive (C), and Tcell intracellular antigen 1-positive (D) intraepithelial lymphocytes were scattered (×200).

\section{DISCUSSION}

EATL is a rare disease in Asia and only a few case series have been reported., ${ }^{2,45}$ The classic EATL is known to be strongly associated with celiac disease. Tumor cells are large and pleomorphic, which are positive for $\mathrm{CD} 3$ and $\mathrm{CD} 7$, but negative for $\mathrm{CD} 4$, CD8, or CD56. EATL type II is mainly composed of small- to medium-sized monomorphic cells, showing immunophenotypic positivity for $\mathrm{CD} 3, \mathrm{CD} 7, \mathrm{CD} 8$, and CD56. It develops sporadically and is independent of celiac disease. ${ }^{6}$ In our cases, CD3, CD8, and CD56 were positive in all patients, while none of them had a history of celiac disease. These results are consistent with another study conducted in Taiwan. ${ }^{7}$

The typical endoscopic features of EATL have scarcely been reported. Recently, there have been a few literatures of EATL type II findings based on double-balloon endoscopy and capsule endoscopy. They reported that diffuse mucosal thickening and edema with multiple shallow ulcerations, and a nodular or 
mosaic mucosal pattern were characteristics of the disease. ${ }^{8-10}$ In our cases, small bowel was almost always involved and colonic involvement was found in half the patients. Endoscopy of the small intestine revealed multiple circular or semicircular shallow ulcerations covered with whitish mucus-like exudate. When the ulcers were encircling the lumen of the small bowel, it was often associated with obstruction. The surrounding mucosa was edematous and thickened by countless fine granularities, showing so-called mosaic mucosal pattern or velvety mucosa These endoscopic features of small bowel may be specific to the disease and thus could be useful for the detection of EATL type II. On the contrary, endoscopy of the colon revealed discrete ulcer with edematous margin or mucosal thickening, sometimes combined with central ulcer. These endoscopic findings of colon are nonspecific to distinguish EATL type II from other disease. Therefore, it may be difficult to diagnose EATL type II only by endoscopic findings of the colon without clinical suspicion.

Patients treated with anthracycline-based chemotherapy regimens had better survival rates than those treated with other forms of therapy (including surgery) or no therapy at all. However, long-term survival is known to be poor even with treatment. According to a retrospective study of 31 patients, the 1and 5-year survival rates were 38.7\% and 19.7\%, respectively. ${ }^{3}$ Another recent study showed that patients treated with highdose chemotherapy followed by APBSCT had a significantly improved survival. It has been estimated that approximately 70\% of all EATL patients may benefit from this therapy. ${ }^{11}$

In summary, we report four cases of EATL type II diagnosed by endoscopy. The endoscopic findings are the mosaic mucosal pattern and diffuse thickening of the mucosa with circumferential shallow ulcerations causing luminal narrowing or obstruction in the small bowel and a discrete ulcer with diffuse mucosal thickening in the colon. EATL mainly involves the small bowel causing diagnostic delay due to the difficulty of access, especially if the symptoms were mild. However, modalities for evaluating the small bowel, such as enteroscopy and capsule endoscopy, have become more developed. Hence, certain typical endoscopic features may give a clue to both clinicians and pathologists in diagnosing EATL in patients with unknown causes for abdominal pain, diarrhea, or bowel obstruction. Currently, there is no treatment guideline for EATL. High-dose chemotherapy followed by autologous stem cell transplantation may be a better treatment option for EATL compared to conventional chemotherapy.

\section{CONFLICTS OF INTEREST}

No potential conflict of interest relevant to this article was reported.

\section{REFERENCES}

1. Swerdlow SH, Campo E, Harris NL, et al. WHO classification of tumours of haematopoietic and lymphoid tissues. Lyon: IARC Press, 2008.

2. Tse E, Gill H, Loong F, et al. Type II enteropathy-associated Tcell lymphoma: a multicenter analysis from the Asia Lymphoma Study Group. Am J Hematol 2012;87:663-668.

3. Gale J, Simmonds PD, Mead GM, Sweetenham JW, Wright DH. Enteropathy-type intestinal T-cell lymphoma: clinical features and treatment of 31 patients in a single center. J Clin Oncol 2000;18: 795-803.

4. Chan JK, Chan AC, Cheuk W, et al. Type II enteropathy-associated T-cell lymphoma: a distinct aggressive lymphoma with frequent gamma delta T-cell receptor expression. Am J Surg Pathol 2011;35:1557-1569.

5. Ko YH, Karnan S, Kim KM, et al. Enteropathy-associated T-cell lymphoma: a clinicopathologic and array comparative genomic hybridization study. Hum Pathol 2010;41:1231-1237.

6. Chott A, Haedicke W, Mosberger I, et al. Most CD56+ intestinal lymphomas are CD8+CD5-T-cell lymphomas of monomorphic small to medium size histology. Am J Pathol 1998;153:1483-1490.

7. Tung CL, Hsieh PP, Chang JH, Chen RS, Chen YJ, Wang JS. Intestinal T-cell and natural killer-cell lymphomas in Taiwan with special emphasis on 2 distinct cellular types: natural killer-like cytotoxic T cell and true natural killer cell. Hum Pathol 2008;39:1018-1025.

8. Yanai S, Matsumoto T, Nakamura S, et al. Endoscopic findings of enteropathy-type T-cell lymphoma. Endoscopy 2007;39 Suppl 1:E339-E340.

9. Sato Y, Ono M, Sagawa T, et al. Endoscopic findings of enteropathy-type T-cell lymphoma by double-balloon enteroscopy and capsule endoscopy. Dig Endosc 2010;22:243-245.

10. Bae JY, Ko BM, Min SK, et al. A case of enteropathy-type T-cell lymphoma diagnosed by small bowel enteroscopy: a perspective on imaging-enhanced endoscopy. Gut Liver 2012;6:516-519.

11. Sieniawski M, Angamuthu N, Boyd K, et al. Evaluation of enteropathy-associated T-cell lymphoma comparing standard therapies with a novel regimen including autologous stem cell transplantation. Blood 2010;115:3664-3670. 